\title{
LISTA DE POLIQUETOS SEDENTARIOS HALLADOS EN EL PERU
}

Juan Tarazona $B$.

Laboratorio de Zoologia General.

Departamento de Ciencias Biológicos.

Universidad Nacional Mayor de San Marcos.

\section{INTRODUCCION}

La elaboración de listas de los diferentes grupos zoológicos posee gran valor, ya que constituyen la base para futuros estudios zoogeogróficos y ecológicos. Tales listas tienen especial importancia en los países de esta parte del Pacífico, donde son muy escasas. En nuestro medio se han realizado algunos avances de este tipo con respecto a los moluscos y crustóceos, pero ninguno con respecto a poliquetos, que conjuntamente con los anteriores, constituyen los grupos más importantes del ecosistema marino.

El conocimiento acerca de los poliquetos del Perú es muy reducido. De poco más de 190 especies de poliquetos registrados hasta el momento, sólo 48 son sedentarios, reportados por diversos autores extranjeros, a partir de 1861 .

Desde hce algún tiempo venimos estudiando nuestra fauna de poliquetos, observando la existencia de varias familias aún sin reportar y que para llegar a conocer el número definitivo de especies se requieren muchos años de ardua labor de colecta y estudios taxonómicos. Por el momento nos parece conveniente publicar esta lista con la que se trata de proporcionar el primer inventario de poliquetos sedentarios del Perú, actualizando y uniformizando la sistemática empleada por los diversos autores, con la finalidad de facilitar y estimular los estudios posteriores. $\mathrm{Si}$ existen omisiones de especies, éstas deben ser muy escasas; pero sí se excluye intencionalmente las determinaciones hasta género de larvas, como las de Polydora y Spio (Ver Berkeley y Berkeley, 1964, póg. 133).

Los nombres de localidades han sido transcritos como aparecen en las publicaciones originales y para la secuencia de las familias se ha seguido el ordenamiento adoptado por Hartman (1959, 1965). Se han omitido las subfamilias. Los gé neros y/especies de cada familia se enumeran en orden alfabético; luego en orden cronológico, los hallazgos en el Perú. Finalmente se ha designado como zona litoral, las citas de: intertidal, orilla $y$ eulitoral.

\section{LISTA SISTEMATICA DE LOS POLIQUETOS SEDENTARIOS HALLADOS EN EL PERU.}

Familia ORBINIIDAE Hartman, 1942.

Naineris brevicephala Hartmann-Schrcder, 1960.

Hartmann-Scroder, 1960, póg. 32. De La Herradura y entre La Herradura y La Chira, en Lima; en zona litoral.

Naineris grubei (Gravier) 1908.

Scoloplos Grubei Gravier, 1908, pág. 42. Paita.

Scoloplos Grubei; Gravier, 1909, pág. 646. Paita.

Naineris laevigata (Grube) 1855. Hartman, 1957, pág. 279. Perú (Colectado por el Dr. Wolfgang Weyrauch).

Protoariciella uncinata Hartmann-Schrœder, 1962.

Hartmann-Schrceder, 1962, pág. 134. Del Km. 235 de la carretera Panamericana Norte; en zona litoral.

Scoloplos armiger trioculata HartmannSchroeder, 1962.

Hartmann-Schroeder, 1962, pág. 134. De Punta Chira, a $30 \mathrm{Km}$. de Camaná; en zona litoral.

\section{Familia PARAONIDAE Cerruti, 1909}

Aedicira brevicornis Hartmann-Schrceder, 1962.

Hartmann-Schrceder, 1962, pág. 135. De Isla Santa y Bahía Coisco, al norte de Chimbote; a $9 \mathrm{~m}$. de profundidad. 
Aedicira punctata Hartmann-Schroeder, 1962.

Hartmann-Schrceder, 1962, pág. 136. De Isla Santa y Bahía Coisco, al norte de Chimbote; a $9 \mathrm{~m}$. de profundidad.

Familia SPIONIDAE Grube, 1850.

Boccardia polybranchia (Haswell) 1885. Polydora euryhalina Hartmann-Schroeder, 1960, pág. 133. De Punta Chira, en Ocoña (sur del Perú), en zona litoral; del río Lurín, $5 \mathrm{Km}$. río arriba, agua dulce.

Polydora sp. 1

Gravier, 1909, pág. 645. De Paita (Perú).

Prionospio ornata Berkeley y Berkeley, 1961.

Berkeley y Berkeley, 1961, pág. 660. Larvas procedentes de Lat. 08 17'S, Long. $79^{\circ} 09^{\prime} \mathrm{W}$; Lat. $08^{\circ} 50^{\prime} \mathrm{S}$, Long. $78^{\circ} 50^{\prime} \mathrm{W}$; Lat. $10^{\circ} 36^{\prime} \mathrm{S}$, Long. $78^{\circ}$ $02^{\prime} \mathrm{W}$. Del plancton.

Berkeley y Berkeley, 1964, pág. 132. Larvas distribuidas entre Lat. $06^{\circ} 31^{\prime} \mathrm{S}$ y $16^{\circ} 18^{\prime}$ 's; del plancton.

Prionospio peruana Hartmann-Schroeder, 1962.

Hartmann-Schrceder, 1962, pág. 138. De Isla Santa y Bahía Coisco, al norte de Chimbote; a una profundidad de $15-18 \mathrm{~m}$.

Prionospio pinnata Ehlers, 1901.

Berkeley y Berkeley, 1964, pág. 132. Larvas distribuidas entre Lat. $06^{\circ} 04^{\prime} \mathrm{S}$ y $06^{\circ} 31^{\prime} \mathrm{S}$; del plancton.

Familia MAGELONIDAE Cunningham y Ramage, 1888.

Magelona annulata Hartmann-Schrœeder, 1962.

Hartmann-Schrœder, 1962, pág. 137. Isla Blanca, en Chimbote (a $8 \mathrm{~m}$. de profundidad); Isla Blanca y Bahía Coisco, al norte de Chimbote (a una profundidad de $15-18 \mathrm{~m}$.).

Magelona phyllisae Jones, 1963. Jones, 1963, póg. 2. De Lat. 09\% 40'S, Long. $79^{\circ} 28^{\prime} \mathrm{W}$; dragado a una profundidad de $181 \mathrm{~m}$.

Familia DISOMIDAE Mesnil, 1897.
Poecilochaetus sp. ?

Berkeley y Berkeley, 1964, pág. 131. Larvas distribuidas entre Lat. $03^{\circ} 33^{\prime} \mathrm{S}$ y $\left.06^{\circ} 4\right)^{\prime} \mathrm{S}$; del plancton.

Familia CHAETOPTERIDAE Malmgren, 1867.

Mesochaetopterus laevis Hartmann-

Schroeder, 1960.

Hartmann-Schroeder, 1960, pág. 35.

Al sur de Bocapón (Lat. 03ㅇ 45's, al norte del Perú); en zona litoral.

Familia CIRRATULIDAE Carus, 1863.

Cauleriella magna-oculata HartmannSchrœder, 1962.

Hartmann-Schrœeder, 1962, pág. 139. De Isla Santa y Bahía Coisco, al norte de Chimbote; a $9 \mathrm{~m}$. de profundidad.

Cirratulus megalus Chamberlin, 1919. Chamberlin, 1919, pág. 375. De Lat. $05^{\circ} 47^{\prime} \mathrm{S}$, Long. $81^{\circ} 24^{\prime} \mathrm{W}$; dragado a 536 brazas de profundidad.

Cirriformia polytricha (Schmarda) 1861. Audouinia polytricha; Gravier, 1909. pág. 641. Paita.

Dodecaceria laddi oculata HartmannSchrceder, 1962.

Hartmann-Schrœeder, 1962, pág. 141. De Punta Chira, a $30 \mathrm{Km}$. al norte de Camaná; a $9 \mathrm{~m}$. de profundidad.

Dodecaceria opulens Gravier, 1908. Gravier, 1908, pág. 41. De Paita, al norte del Perú.

Gravier, 1909, pág. 643. De Paita, al norte del Perú.

Tharyx aequiseta Hartmann-Schroeder, 1962.

Hartmann-Schrceder, 1962, póg. 139. De Isla Santa y Bahía Coisco, al norte de Chimbote; a $9 \mathrm{~m}$. de profundidad.

Tharyx sp.

Heterocirrus sp. 1 Gravier, 1909, póg. 642. De Paita (Perú).

Familia COSSURIDAE Day, 1963.

Cossura laeviseta Hartmann-Schrœeder, 1962.

Hartmann-Schrceder, 1962, póg. 140. De Isla Santa y Bahía Coisco, al norte de Chimbote; a $9 \mathrm{~m}$. de profundidad. Familia FLABELLIGERIDAE Saint-Joseph, 1894 . 
Ilyphagus pluto Chamberlin, 1919. Chamberlin, 1919, póg. 403. De Lat. $13^{\circ} 11^{\prime} \mathrm{S}$, Long. $78^{\circ} 18^{\prime} \mathrm{W}$; a 2,895 brazas de profundidad.

Pherusa capensis (Mc Intosh) 1885. Stylarioides (Throphonia) capensis; Gravier, 1909, pág. 649. De Paita (Perú). Familia OPHELIIDAE Malmgren, 1867.

Travisia elongata Grube, 1866. Grube, 1866, pág. 66. De Samoa, pero Ehlers, 1901, pág. 171 corrige a lquique, Perú (actualmente en Chile).

Travisia profundi Chamberlin, 1919. Chamberlin, 1919, pág. 387. De Lat. $05^{\circ} 42^{\prime} \mathrm{S}$, Long. $83^{\circ} \mathrm{W} ;$ a 2,222 brazas de profundidad.

Familia CAPITELLIDAE Grube, 1862.

Mediomastus branchiferus HartmannSchrœder, 1962.

Hartmann-Schroder, 1962, pág. 142. Del Callao, entre La Punta y El Camotal; Isla Santa y Bahía Coisco, al norte de Chimbote. En rizoides de Macrocystis.

Parheteromastides multioculatus Hart* mann-Schrceder, 1962.

Hartmann-Schrœeder, 1962, pág. 144. De Isla Santa y Bahía Coisco, al norte de Chimbote; a $9 \mathrm{~m}$. de profundidad.

Familia ARENICOLIDAE Johnston, 1846.

Arenicola glacialis Murdoch, 1885 peruana Hartman-Schrœeder, 1960.

Hartmann-Schroeder, 1960, póg. 37. De Las Carpitas, en Mancora (al norte del Perú).

Familia MALDANIDAE Malmgren, 1867.

Sonatsa meridionalis Chamberlin, 1919. Chamberlin, 1919, pág. 416. De Lat. $05^{\circ} 42^{\prime} \mathrm{S}$, Long. $83^{\circ} \mathrm{W}$; a 2,222 brazas de profundidad.

Familia SABELLARIIDAE Johnston, 1865.

Phragmatopoma lapidosa Kinberg, 1867. Sabellaria Fauvelli Gravier, 1908, pág. 43. De Paita (Perú).

Sabellaria Fauvelli; Gravier, 1909, póg 650. De Paita (Perú).
Phragmatopoma moerchi Kinberg, 1867. Posiblemente como Hermella orbifera Ehlers, 1901, pág. 267. Del Perú (Fide Hartman, 1959, pág. 471)

Hartman, 1944, pág. 350. De Bahía de la Independencia, a 10 brazas de profundidad; al sur de Zorritos, en zona litoral.

Hartmann-Schrœeder, 1960, pág. 38. De La Herradura, en Lima; Cancas y sur de Bocapán, al norte del Perú. En zona litoral.

Phragmatopoma peruensis Hartman, 1944.

Hartman, 1944, póg. 353. De Salaverry; en zona litoral.

Sabellaria bella Grube, 1870 . Hartman, 1944, pág. 342. De Bahía de la Independencia; en zona litoral.

Familia AMPHARETIDAE Malmgren, 1867.

Moyanus explorans Chamberlin, 1919. Chamberlin, 1919, pág. 452. De Lat. $05^{\circ} 42^{\prime} \mathrm{S}$, Long. $83^{\circ} \mathrm{W}$; a 2,222 brazas de profundidad.

Paiwa abyssi Chamberlin, 1919.

Chamberlin, 1919, pág. 459. De Lat. $05^{\circ} 17^{\prime} \mathrm{S}$, Long. $85^{\circ} 20^{\prime} \mathrm{W} ;$ a 2,235 brazas de profundidad.

Familia TEREBELLIDAE Malmgren, 1867.

Nicolea profundi Chamberlin, 1919. Chamberlin, 1919, pág. 429. De Lat. $05^{\circ} 46^{\prime} \mathrm{S}$, Long. $81^{\circ} 31^{\prime} 09^{\prime \prime} \mathrm{W}$; a 1,036 brazas de profundidad.

Nicolea zostericola (Oersted) 1844. Berkeley y Berkeley, 1961, pág. 662. Postlarvas procedentes de Lat. $05^{\circ} 55^{\prime} \mathrm{S}$ Long. $82^{\circ} 20^{\prime} \mathrm{W}$; del plancton.

Pseudoampharete tentaculata HartmannSchroeder, 1960.

Hartmann-Schrœeder, 1960, pág. 39, como AMPHARETIDAE. Puede ser referible a TEREBELLIDAE (Fide Hartman, 1965, pág. 72). De cerca a Can-

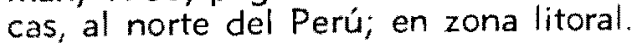

Familia TRICHOBRANCHIDAE Malmgren, 1866 
Terebellides eurystethus Chamberlin, 1919.

Chamberlin, 1919, pág. 438. De Lat. $11^{\circ} 55^{\prime} \mathrm{S}$, Long. $81^{\circ} 20^{\prime} \mathrm{W}$; a 2,600 brazas de profundidad. Tal vez Terebellides stroemi (Fide Hartman, 1959, póg. 533).

Familia SABELLIDAE Malmgren, 1867.

Chone paracincta Hartmann-Schroeder, 1962.

Hartmann-Schrceder, 1962, pág. 145.

Del Callao, entre La Punta y El Camotal (a $3 \mathrm{~m}$. de profundidad); Isla Santa y Bahía Coisco, al norte de Chimbote (a una profundidad de 15 a $18 \mathrm{~m}$.).

Demonax leucaspis Kinberg, 1867. Kinberg, 1867, pág. 354. De la Isla San Lorenzo (Perú).

Megalomma roulei (Gravier) 1907. Branchiomma Roulei Gravier, 1907, pág. 526. De Paita (Perú).

Branchiomma Roulei; Gravier, 1908, pág. 44. De Paita (Perú).

Branchiomma Roulei; Gravier, 1909, pág. 655. De Paita (Perú).

Branchiomma roulei; Berkeley y Berkeley, 1961, pág. 662. De Mancora (Perú); en zona litoral.

Potamilla anophthalma Hartmann-Schrceder, 1960.

Hartmann-Schroeder, 1960, póg. 41. De cerca a Cancas, al norte del Perú; en zona litoral.

Potamilla modesta (Quatrefages) 1865. Sabella modesta Quatrefages, 1865, póg. 451. De Lima (Perú).

Sabella tilosaula Schmarda, 1861. Schmarda, 1861, pág. 34. Del Perú. Tal vez Hypsicomus phaeotaenia (Fide Hartman, 1959, pág. 565).

\section{REFERENCIAS BIBLIOGRAFICAS}

BERKELEY, E. y BERKELEY, C. - 1961. Notes on Polychaeta from California to Peru. Can. J. Zool. 39:655-664.

BERKELEY, E. y BERKELEY, C. - 1964. Notes on some pelagic and some swarming Polychaeto taken off the coost of Peru. Ibid. 42:121-134.

CHAMBERLIN, R. V. - 1919. The Anmelido Polychoeto. Mus. Comp. Zool. Harvard, Mem. 48: 1.514 .

GRAVIER, Ch. - 1909. Annélides polychétes recueillies á Payta (Pérou) par $M$. le Dr. Rivet. Arch. Zool. Exp. Gén., ser. T. 10:617-659, p). 16-18.

HARTMAN, Olga - 1944. Polychoetous Annelids. Pt. VI: Paroonidae, Magelonidae, Longosomidae, Ctenodrilidae and Sabelloriidoe. Allan Hancock Pacific Exped. 10:311-389, pl. $27-42$

HARTMAN, Olga - 1957. Orbiniidoe, Apistobranchidae, Paraonidae, and Longosomidae. lbid. 15:2| $\mid-394$, pl. 20-44, 1 chart,

HARTMAN, Olgo - 1959. Cotalogue of the Polychaetous Annelids of the World. Part 11. Allan Hancock Fdn. Occ. Papers, 23:355628.

HARTMAN, Olgo - 1965. Catalogue of the Polychaetous Annelids of the World. Supplement 1960-1965 and index. Ibid. 23:1-197.

HARTMANN-SCHRCEDER, G. - 1960. Zur Polychaeten-Founa von Peru. Beitr. Zur Neotrop. Fauna, 2:1-44, abb. 1-92.

HARTMANN SCHRCEDER, G. - 1962. Zweiter Beitrag zur Polychaetenfauno von Peru. Kieler Meresforsch. 18:109-147, tafel 1-20, abb. 1-127.

JONES, Meredith L. - 1963. Four New Species of Magelono (Annelida, Polychaeto) and a Redescription of Magelona longicornis Johnson. Amer. Mus. Novitat. 2164:1-31, fig. 1.70 . 